\title{
On Global Bifurcations of Three-dimensional Diffeomorphisms Leading to Lorenz-like Attractors
}

\author{
S.V. Gonchenko ${ }^{1}$, I.I. Ovsyannikov ${ }^{12 *}$ \\ ${ }^{1}$ Research Institute of Applied Mathematics and Cybernetics, \\ 10, Ulyanova Str. 603005 Nizhny Novgorod, Russia \\ ${ }^{2}$ Imperial College, SW7 2AZ London, UK
}

\begin{abstract}
We study dynamics and bifurcations of three-dimensional diffeomorphisms with nontransversal heteroclinic cycles. We show that bifurcations under consideration lead to the birth of Lorenz-like attractors. They can be viewed as attractors in the Poincare map for periodically perturbed classical Lorenz attractors and hence they can allow for the existence of homoclinic tangencies and wild hyperbolic sets.
\end{abstract}

Keywords and phrases: homoclinic and heteroclinic orbits, bifurcations, strange attractors, saddle-focus

Mathematics Subject Classification: 37C05, 37C29, 37G25, 37G35

\section{Introduction}

We study bifurcations of a three-dimensional diffeomorphism $F_{0}$ with a nontransversal heteroclinic cycle that consists of two fixed points $O_{1}$ and $O_{2}$ and two heteroclinic orbits $\Gamma_{12} \subset W^{u}\left(O_{1}\right) \cap W^{s}\left(O_{2}\right)$ and $\Gamma_{21} \subset W^{u}\left(O_{2}\right) \cap W^{s}\left(O_{1}\right)$. We assume that the points $O_{1}$ and $O_{2}$ are both saddle-foci with multipliers $\lambda_{1} e^{ \pm i \varphi}, \gamma_{1}$ for $O_{1}$ and $\lambda_{2} e^{ \pm i \psi}, \gamma_{2}$ for $O_{2}$ such that $0<\lambda_{i}<1<\gamma_{i}, i=1,2$ and $\varphi, \psi \in(0, \pi)$. We consider the case when exactly one of the heteroclinic orbits is nontransversal, let it be $\Gamma_{21}$. Thus we assume that $W^{u}\left(O_{1}\right)$ and $W^{s}\left(O_{2}\right)$ intersect transversely at the points of $\Gamma_{12}$, and $W^{u}\left(O_{2}\right)$ and $W^{s}\left(O_{1}\right)$ have a quadratic tangency at the points of $\Gamma_{21}$, see Fig. 1. We make an important additional assumption that the Jacobian $J$ of $F_{0}$ is greater than 1 at one fixed point and is less than 1 at the other or in other words the cycle is of the mixed type [1]. Namely we consider the case where $J\left(O_{1}\right)=\lambda_{1}^{2} \gamma_{1}<1$ and $J\left(O_{2}\right)=\lambda_{2}^{2} \gamma_{2}>1$ (the result remains the same in the case $J\left(O_{1}\right)>1$ and $J\left(O_{2}\right)<1$ as well).

Historically the study of bifurcations of nontransversal heteroclinic cycles went in parallel with the study of homoclinic bifurcations since these types of bifurcations have much in common. In particular, the first paper on the nontransversal heteroclinic cycle (contour) by Gavrilov [2] was published at the same time with the first paper on homoclinic tangencies by Gavrilov and Shilnikov [3]. Comparing these papers, we find many similarities in dynamical properties and bifurcations.

The situation was drastically changed after the paper [4] where bifurcations of two-dimensional diffeomorphisms with nontransversal heteroclinic cycles were studied in the case where the Jacobians at two

\footnotetext{
${ }^{*}$ Corresponding author. E-mail: Ivan.I.Ovsyannikov@gmail.com
} 
saddle points are greater than one and less than one respectively. As it was shown in [4], bifurcations of such cycles lead to mixed dynamics. Namely near each such diffeomorphism in the space of dynamical systems there are certain open domains (Newhouse regions in fact), where generic systems with the following properties compose a residual set:

1) every system has infinitely many coexisting stable, completely unstable and saddle periodic orbits (as well as stable and unstable closed invariant curves $[1,5])$;

2) the closure of the set of the stable periodic orbits has a nonempty intersection with the closure of the set of the completely unstable ones. Moreover this intersection can contain a nontrivial transitive uniformly hyperbolic set $[6,7]$.

These results have found an application in the study of reversible two-dimensional maps where heteroclinic cycles of mixed type emerge naturally. Following this, one may speak about a phenomenon of a reversible mixed dynamics, $[8,9]$, when in the corresponding Newhouse domains generic reversible maps have infinitely many attracting, repelling, saddle and also elliptic periodic orbits. It is well-known that reversible systems are frequent in applications. In particular, many models from the rigid body dynamics are of this type ${ }^{1}$. We think that the mixed dynamics phenomenon and in particular, the reversible mixed dynamics should be considered as a new important kind of the dynamical chaos.

Note that the mixed dynamics can occur in systems of dimension 2 or higher for diffeomorphisms and 3 or higher for flows. The first examples of systems near which the Newhouse regions with mixed dynamics exist were given in the paper [11] by D. Turaev. In particular, two classes of heteroclinic cycles of mixed type were presented there, for even and odd dimensions of the phase space. In both cases the cycles contained two fixed points $O_{1}$ and $O_{2}$ with the Jacobians less than 1 in one point and greater than 1 in another, and two heteroclinic orbits one of which is nontransversal (a quadratic tangency). If the dimension is even, then the fixed points have the following sets of multipliers:

$$
O_{1}: \quad \lambda_{1}, \lambda_{2} e^{ \pm i \varphi_{2}}, \ldots, \lambda_{n} e^{ \pm i \varphi_{n}}, \gamma_{1} \text { and } O_{2}: \nu_{1} e^{ \pm i \psi_{1}}, \ldots, \nu_{n-1} e^{ \pm i \psi_{n-1}}, \nu_{n}, \gamma_{2},
$$

and if the dimension is odd, then the fixed points have the sets of multipliers:

$$
O_{1}: \lambda_{1} e^{ \pm i \varphi_{1}}, \ldots, \lambda_{n} e^{ \pm i \varphi_{n}}, \gamma_{1} \text { and } O_{2}: \nu_{1}, \nu_{2} e^{ \pm i \psi_{2}}, \ldots, \nu_{n} e^{ \pm i \psi_{n}}, \nu_{n+1}, \gamma_{2},
$$

where $\left|\gamma_{1}\right|>1>\left|\lambda_{1}\right|>\cdots>\left|\lambda_{n}\right|$ and $\left|\gamma_{2}\right|>1>\left|\nu_{1}\right|>\cdots>\left|\nu_{n}\right|\left(>\left|\nu_{n+1}\right|\right)$.

The main idea of this choice of the spectra is that it prevents the diffeomorphism from having a smooth invariant manifold (containing points $O_{1}$ and $O_{2}$ as well as the heteroclinic orbits) whose dimension would be smaller than the dimension $d$ of the phase space. This property along with the condition on Jacobians means that the so-called effective dimension of the problem, [11], is equal to $d$. It implies, by [11], that periodic orbits with $d$ multipliers on the unit circle can be born after certain bifurcations of such cycles and the phenomenon of mixed dynamics will take place for the corresponding Newhouse regions. We will call such heteroclinic cycles the cycles of Turaev type.

Note that such cycles also can exist in the reversible case, where $O_{1}$ and $O_{2}$ are symmetric to each other (in particular, if $\lambda$ is a multiplier of $O_{1}$, then $\lambda^{-1}$ is a multiplier of $O_{2}$ ). For example, in the case where $\operatorname{dim} W^{s}\left(O_{i}\right)=\operatorname{dim} W^{u}\left(O_{i}\right)=m, i=1,2$, one can consider such sets of the multipliers:

$$
\begin{array}{ll}
O_{1}: & \lambda_{0}, \lambda_{1} e^{ \pm i \varphi_{1}}, \ldots, \lambda_{k} e^{ \pm i \varphi_{k}} ; \gamma_{1} e^{ \pm i \psi_{1}}, \ldots, \gamma_{k} e^{ \pm i \psi_{k}}, \gamma_{0} \\
O_{2}: & \gamma_{1}^{-1} e^{ \pm i \psi_{1}}, \ldots, \gamma_{k}^{-1} e^{ \pm i \psi_{k}}, \gamma_{0}^{-1}, \lambda_{0}^{-1} ; \lambda_{1}^{-1} e^{ \pm i \varphi_{1}}, \ldots, \lambda_{k}^{-1} e^{ \pm i \varphi_{k}}
\end{array}
$$

if $m=2 k+1$ and

$$
\begin{array}{ll}
O_{1}: & \lambda_{1} e^{ \pm i \varphi_{1}}, \ldots, \lambda_{k} e^{ \pm i \varphi_{k}} ; \gamma_{0}, \gamma_{1} e^{ \pm i \psi_{1}}, \ldots, \gamma_{k-1} e^{ \pm i \psi_{k-1}}, \gamma_{k} \\
O_{2}: & \gamma_{0}^{-1}, \gamma_{1}^{-1} e^{ \pm i \psi_{1}}, \ldots, \gamma_{k-1}^{-1} e^{ \pm i \psi_{k-1}}, \gamma_{k}^{-1} ; \lambda_{1}^{-1} e^{ \pm i \varphi_{1}}, \ldots, \lambda_{k}^{-1} e^{ \pm i \varphi_{k}}
\end{array}
$$

\footnotetext{
${ }^{1}$ For example, mixed dynamics is one of principal types of orbit behaviour in mechanical nonholonomic models. Thus, it was shown in [10] that, for a model of celtic stone, one of the types of chaotic orbit behaviour is related to the reversible mixed dynamics.
} 
if $m=2 k$.

Bifurcations of three-dimensional nontransversal heteroclinic cycles of Turaev type were studied in the paper [12]. Namely the case was considered where the point $O_{1}$ is a saddle-focus with multipliers $\lambda e^{ \pm i \varphi}, \gamma_{1}$, where $0<\lambda<1<\left|\gamma_{1}\right|$, and the point $O_{2}$ is a saddle with multipliers $\lambda_{1}, \lambda_{2}, \gamma_{2}$ where $0<\left|\lambda_{2}\right|<\left|\lambda_{1}\right|<1<\left|\gamma_{2}\right|$. The main result of [12] was a proof of the birth of strange Lorenz-like attractors in three parameter families of diffeomorphisms which unfold the heteroclinic tangency generically. In fact, this problem was initiated by papers [13], in which the existence of Lorenz-like attractors in 3D Hénon maps was shown, and [14], in which 3D Hénon maps were constructed as rescaled normal forms of first return maps near a homoclinic tangency to a saddle-focus fixed point.

Note that the above mentioned Lorenz-like attractors for diffeomorphisms can be viewed as attractors in the Poincare map for a flow perturbed by a periodic force and having the Lorenz attractor. Therefore such attractor can be wild and, in contrast to the classical Lorenz attractor, it can allow homoclinic tangencies and hence be a wild hyperbolic attractor [15], [16]. In other words the diffeomorphism itself, as well as the close ones, belongs to some Newhouse domain but stable periodic orbits do not appear here due to the fact that the periodically perturbed attractor inherits the property of pseudo-hyperbolicity from the Lorenz attractor in the flow [15].

Concerning the definition of the Lorenz-like attractor for diffeomorphisms please refer to the paper [17] in the present issue.

In this paper we have the same goal as in [14]: to study global bifurcations of 3D diffeomorphisms leading to the appearance of Lorenz-like strange attractors. However we consider here a 3D diffeomorphism with a nontransversal heteroclinic cycle which is not of Turaev type. Namely, we assume that the cycle contains two fixed points $O_{1}$ and $O_{2}$ which are both saddle-foci and the Jacobian of the diffeomorphism is less that 1 at one fixed point and is greater than 1 in another.

Section 2 contains the statement of the problem and formulation of main results including Theorems 2.1 and 2.2. We note that Theorem 2.2 is deduced from Theorem 2.1 and the corresponding deduction (proof of Theorem 2.2) is contained in Section 2. Birth of Lorenz-like attractors in 3D maps is discussed in Section 3. We perform the proof of Theorem 2.1 in Section 4.

\section{Statement of the problem and the main results}

Consider an orientation-preserving three-dimensional diffeomorphism $F_{0} \in C^{r}, r \geq 4$, which satisfies the following conditions (see Fig. 1):

(A) $\quad F_{0}$ has two fixed points: a saddle-focus $O_{1}$ with multipliers $\left(\lambda_{1} e^{i \varphi}, \lambda_{1} e^{-i \varphi}, \gamma_{1}\right)$ where $0<\lambda_{1}<1<$ $\gamma_{1}, \varphi \in(0, \pi)$, and a saddle-focus $O_{2}$ with multipliers $\left(\lambda_{2} e^{i \psi}, \lambda_{2} e^{-i \psi}, \gamma_{2}\right)$ such that $0<\lambda_{2}<1<\gamma_{2}$, $\psi \in(0, \pi)$

(B) At one of the fixed points the Jacobian of the map is less than 1 in the absolute value, and it is greater than 1 at the other fixed point.

(C) The invariant manifolds $W^{u}\left(O_{1}\right)$ and $W^{s}\left(O_{2}\right)$ intersect transversely at the points of a heteroclinic orbit $\Gamma_{12}$; the invariant manifolds $W^{u}\left(O_{2}\right)$ and $W^{s}\left(O_{1}\right)$ have a quadratic tangency at the points of a heteroclinic orbit $\Gamma_{21}$.

We stress that according to the condition (B) the diffeomorphism contracts volume near one of the fixed points and expands volume near the other point. The flows of systems of differential equations with this property are usually called the flows with alternating divergence. Following [1], we call such diffeomorphisms and the heteroclinic cycle $C=\left\{O_{1}, O_{2}, \Gamma_{12}, \Gamma_{21}\right\}$ of mixed type (the term "contractingexpanding" from [12] seems also quite suitable).

Diffeomorphisms that are close to $F_{0}$ and satisfy the condition $(\mathrm{C})$ (note that the conditions (A) and (B) are the non-degeneracy conditions and are valid for all close systems) comprise, in the space of $C^{r}$-diffeomorphisms, a codimension-one bifurcation surface $\mathcal{H}_{\text {het }}$.

Let $U$ be a sufficiently small and fixed neighborhood of the heteroclinic cycle $C=\left\{O_{1}, O_{2}, \Gamma_{12}, \Gamma_{21}\right\}$. It can be represented as a union of small neighborhoods $U_{1}$ of $O_{1}$ and $U_{2}$ of $O_{2}$ with a (finite) number of small neighborhoods of those points of the heteroclinic orbits $\Gamma_{12}$ and $\Gamma_{21}$ which do not belong to $U_{1} \cup U_{2}$. 


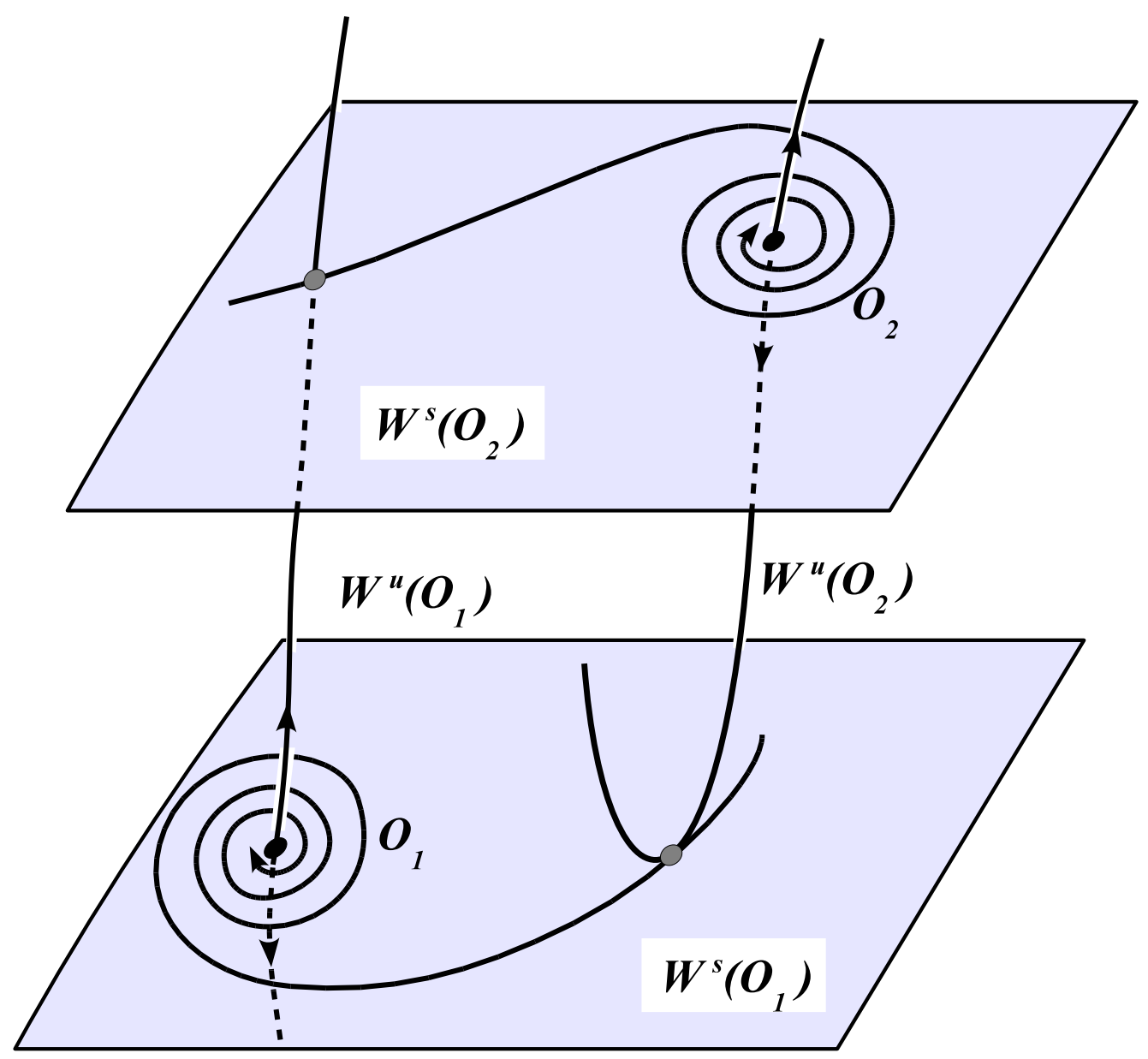

FigURE 1. A heteroclinic cycle in the map $F_{0}$ under assumptions A-C.

We will investigate bifurcations of single-round periodic orbits lying entirely in $U$. Every single point of such orbit can be considered as a fixed point of the corresponding first return map.

Prior to the investigation of the bifurcations themselves we need to establish what parameters $\varepsilon$ should be taken as governing. The number of them can be chosen, according to [11], equal to the so-called effective dimension of the problem which coincides with the number of zero Lyapunov exponents that may appear for the trajectories of the close systems. In our case this number is 3 as the contour contains saddle-foci (so that, in contrast with the case regarding saddles [18], here a smooth global two-dimensional center manifold does not exist) and also due to the condition (B) the three-dimensional volumes can both shrink and expand. Therefore for a reasonable study of the bifurcations the number of the governing parameters should be not less than 3 . In the present paper we will consider three-parametric families (general unfoldings) $F_{\varepsilon}$, where $\varepsilon=\left(\varepsilon_{1}, \varepsilon_{2}, \varepsilon_{3}\right)$.

It is natural to choose the splitting parameter $\mu$ for the quadratic heteroclinic tangency with respect to one of the points of $\Gamma_{21}$ as the first governing parameter $\varepsilon_{1}$. 
Note that the diffeomorphisms on $\mathcal{H}_{\text {het }}$ possess $\Omega$-moduli i.e. continuous topology conjugacy invariants on the set of non-wandering orbits [19], [20]. By the definition of an $\Omega$-modulus any change of its value leads to a bifurcation on the set of non-wandering orbits (periodic, homoclinic etc). This means that the $\Omega$-moduli are also the natural bifurcation parameters and they should be included into the set of the governing parameters if necessary. In our case the most important $\Omega$-moduli are the angle arguments of the multipliers of the fixed points $O_{1}$ and $O_{2}, \varphi$ and $\psi$ correspondingly. We take one of them as the second governing parameter $\varepsilon_{2}$, in the following way:

$$
\varepsilon_{2}=\varphi(\varepsilon)-\varphi(0) .
$$

Finally note that as we are dealing with the contracting-expanding (mixed) case we need one more parameter $\varepsilon_{3}$ that will control the values of the Jacobians $J_{1}=\lambda_{1}^{2} \gamma_{1}$ and $J_{2}=\lambda_{2}^{2} \gamma_{2}$ in the fixed points $O_{1}$ and $O_{2}$ of the map $F_{\varepsilon}$. As it will be shown later, the following parameter can effectively play this role:

$$
\varepsilon_{3}=S\left(F_{\varepsilon}\right)-S\left(F_{0}\right)
$$

where $\mathrm{S}(\mathrm{F})$ is a functional defined as $S(F)=-\frac{\ln J_{1}}{\ln J_{2}}$.

The following Theorem states the form the first return maps can be brought to:

Theorem 2.1. Let $F_{\varepsilon}$ be the three-parameter family defined above. Then the point $\varepsilon=0$ in the space of parameters is a limit of an infinite sequence of open domains, $\Delta_{i j}$, such that for $\varepsilon \in \Delta_{i j}$ the first return map of a certain open region $V_{i j} \subset U$ using smooth changes of the coordinates and the parameters can be brought to the following form

$$
\begin{aligned}
& \bar{X}=Y+o(1)_{i, j \rightarrow+\infty}, \quad \bar{Y}=Z+o(1)_{i, j \rightarrow+\infty}, \\
& \bar{Z}=M_{1}+B X+M_{2} Y-Z^{2}+o(1)_{i, j \rightarrow+\infty},
\end{aligned}
$$

where $(X, Y, Z)$ are coordinates in $V_{i j}$ whose ranges cover all finite values as $i, j \rightarrow \infty$, parameters $\left(M_{1}, M_{2}, B\right)$ are related to $\varepsilon$ by a diffeomorphism, and as $\varepsilon$ runs over $\Delta_{i j}$ the values of $\left(M_{1}, M_{2}, B\right)$ run over regions that cover all finite values of $\left(M_{1}, M_{2}\right)$ and all finite positive values of $B$ as $i, j \rightarrow \infty$. The $o(1)$-terms are functions of $\left(X, Y, Z, M_{1}, M_{2}, B\right)$ that tend to zero as $i, j \rightarrow \infty$, along with all derivatives up to the order $(r-2)$.

The geometry of the action of the first return map

$$
T_{i j}=T_{21} T_{02}^{j} T_{12} T_{01}^{i}: V_{i j} \subset \Pi_{1}^{+} \rightarrow \Pi_{1}^{-} \rightarrow \Pi_{2}^{+} \rightarrow \Pi_{2}^{-} \rightarrow \Pi_{1}^{+}
$$

is illustrated in Fig. 2. Here $M_{1}^{-}, M_{2}^{+}$and $M_{2}^{-}, M_{1}^{+}$are some points of orbits $\Gamma_{12}$ and $\Gamma_{21}$ correspondingly, taken at $\varepsilon=0$, moreover there exist some integers $q_{1}$ and $q_{2}$ for which $M_{2}^{+}=F_{0}^{q_{1}}\left(M_{1}^{-}\right)$and $M_{1}^{+}=$ $F_{0}^{q_{2}}\left(M_{1}^{-}\right)$, also $\Pi_{s}^{+} \subset U_{s}, \Pi_{s}^{-} \subset U_{s}, s=1,2$, are certain small neighbourhoods of these points. $T_{0 s}$ is a so-called local map, defined as a restriction of the diffeomorphism $F_{\varepsilon}$ onto the neighborhood of the point $O_{s}$, i.e. $\left.T_{0 s} \equiv F_{\varepsilon}\right|_{U_{s}}$. The global maps $T_{12}: \Pi_{1}^{-} \rightarrow \Pi_{2}^{+}$and $T_{21}: \Pi_{2}^{-} \rightarrow \Pi_{1}^{+}$are defined as $T_{12}=\left.F_{\varepsilon}^{q_{1}}\right|_{\Pi_{1}^{-}}$ and $T_{21}=\left.F_{\varepsilon}^{q_{2}}\right|_{\Pi_{2}^{-}}$. For more details and the exact formulas see Section 4 .

We would like to mention that for large $i$ and $j$ the following relationships between the initial and the final parameters hold (see also the exact formulas (4.11), (4.17) and (4.18) below):

$$
\begin{aligned}
& M_{1} \sim \gamma_{1}^{2 i} \gamma_{2}^{2 j}\left(\mu+O\left(\lambda_{2}^{j}+\gamma_{1}^{-i}\right)\right) \\
& M_{2} \sim\left(\lambda_{1} \gamma_{1}\right)^{i}\left(\lambda_{2} \gamma_{2}\right)^{j} \cos \left(i \varphi+\beta_{1}\right) \cos \left(j \psi+\beta_{2}\right), \\
& B \sim\left(\lambda_{1}^{2} \gamma_{1}\right)^{i}\left(\lambda_{2}^{2} \gamma_{2}\right)^{j} \equiv J_{1}(\varepsilon)^{i} J_{2}(\varepsilon)^{j}
\end{aligned}
$$

where $\beta_{1}$ and $\beta_{2}$ are some constants (invariants of the diffeomorphism $F_{0}$ ). The domains $\Delta_{i j}$ from the Theorem 2.1 correspond to those values of the initial parameters $\varepsilon$ for which the rescaled parameters $M_{1}$, $M_{2}$ and $B$ take finite values (say, $\left\|\left(M_{1}, M_{2}, B\right)\right\| \leq L$ for some pre-defined positive constant $L$ ). It is 


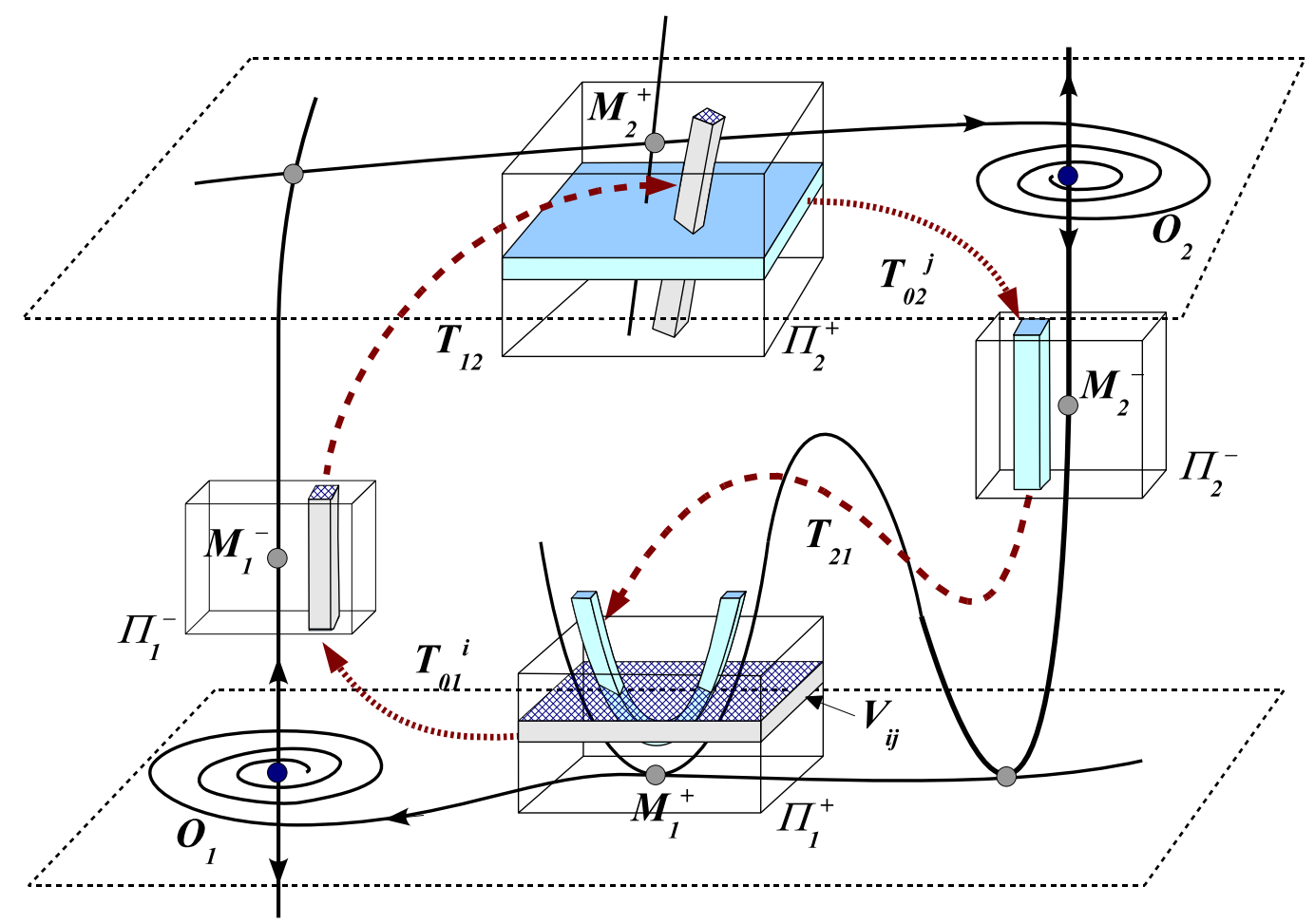

FIGURE 2. Geometry of the local and global maps $T_{01}, T_{02}$ and $T_{12}, T_{21}$, respectively, for the diffeomorphism $F_{\varepsilon}$.

obvious that for large $i$ and $j$, one can obtain arbitrary finite values of the parameters $M_{1}$ and $M_{2}$ via arbitrary small independent variations of the parameters $\mu$ and $\varphi$ correspondingly (or $\mu$ and $\psi$ ). It is possible because the values $\gamma_{1}^{2 i} \gamma_{2}^{2 j}$ and $\left(\lambda_{1} \gamma_{1}\right)^{i}\left(\lambda_{1} \gamma_{2}\right)^{j} \sim B \lambda_{1}^{-i} \lambda_{2}^{-j}$ are asymptotically large as $i, j \rightarrow \infty$. The same refers to the parameter $B$. Indeed as $J_{1}$ and $J_{2}$ are positive we can take a logarithm from the both sides of the last equality from (2.4). As a result we obtain $\ln B \sim i \ln J_{1}+j \ln J_{2}$. By virtue of (2.1) this relationship can be rewritten as

$$
\frac{\ln B}{\ln \left|J_{2}\right|} \sim j-i\left(\varepsilon_{3}-S\left(F_{0}\right)\right) .
$$

Provided that $S\left(F_{0}\right)=\ln \left|J_{1}(0)\right| / \ln \left|J_{2}(0)\right|<0$ by the condition (B), the relationship (2.5) can be always satisfied while varying the parameter $\varepsilon_{3}$ value for arbitrary large $i$ and $j$. Note that we should take some particular infinite sequence of integer $i$ for which the value of $\cos \left(i \varphi+\beta_{1}\right)$ remains asymptotically small.

Omitting the $o(1)$ in $(2.2)$ we obtain the following 3D Henon map:

$$
\bar{x}=y, \quad \bar{y}=z, \quad \bar{z}=M_{1}+B x+M_{2} y-z^{2},
$$

The Theorem 2.1 shows that the map (2.6) appears to be the normal form for the corresponding first return maps. Due to this we are able to study bifurcations of single-round periodic orbits in the family $F_{\varepsilon}$ for $\varepsilon \in \Delta_{i j}$ using the standard local analysis of bifurcations of the fixed points of the quadratic map (2.6). Here one can obtain various results - particularly such as the Theorem of a cascade of Lorenz-like attractors for diffeomorphisms analogous to the one from [14]: 
In the parameter space $\varepsilon$ in any neighbourhood of the origin $\varepsilon=0$ there exist a countable set of open domains $\delta_{i j} \subset \Delta_{i j}$ such that the diffeomorphism $F_{\varepsilon}$ for $\varepsilon \in \delta_{i j}$ possesses a (local) Lorenz-like attractor ${ }^{2}$.

Indeed, this result immediately follows from the Theorem 2.1 because 1 ) the Lorenz-like attractor exists in the map (2.6) for the parameters values $\left(M_{1}, M_{2}, B\right)$ from some open domain, see [13], [17] and Section 3, and 2) such attractors are robust with respect to perturbations of the class $C^{2}$, [16]. However here it is possible to obtain a stronger result: existence of a countable set of Lorenz-like attractors for diffeomorphisms:

Theorem 2.2. In every neighbourhood of the point $\varepsilon=0$ in the parameter space there exist open domains $\mathcal{N}$ in which those values of $\varepsilon$ are dense for which the corresponding diffeomorphism $F_{\varepsilon}$ possesses a countable set of co-existing Lorenz-like attractors.

Proof. Note that the domains $\mathcal{N}$ from the statement of the Theorem are essentially some Newhouse regions: by definition, the parameter values corresponding to existence of homoclinic tangencies are dense within such domains. But in the case under consideration the domains $\mathcal{N}$ possess some specific feature. Namely, as follows from [21] and [4], in every neighbourhood of the point $\varepsilon=0$ in the parameter space there exist such Newhouse regions $\mathcal{N}$ in which the systems having a non-transversal heteroclinic cycle of the initial kind (i.e. satisfying the conditions (A)-(C)) are dense. Moreover, new points (fixed or periodic) $O_{1}(\varepsilon), O_{2}(\varepsilon)$ and a new orbit $\Gamma_{12}(\varepsilon)$ will be close to those from the initial diffeomorphism $F_{0}$; however a new non-transversal heteroclinic orbit $\tilde{\Gamma}_{12}(\varepsilon)$ (along which the manifolds $W^{s}\left(O_{1}(\varepsilon)\right.$ ) and $W^{u}\left(O_{2}(\varepsilon)\right.$ ) have a quadratic tangency) despite it entirely lies within $U$, will not be close to $\Gamma_{21}$ (with respect to $\Gamma_{21}$ it will be multi-round). Now the proof of the Theorem can be performed employing the method of the embedded domains. Indeed, it follows from the Theorem 2.1 that near any value of $\varepsilon \in \mathcal{N}$ for which $F_{\varepsilon}$ has a non-transversal heteroclinic cycle there exists an open domain $u_{1}$ of the parameters values corresponding to existence of a Lorenz-like attractor. Since $u_{1}$ belongs to $\mathcal{N}$ there exist other values of $\varepsilon$ corresponding to a new non-transversal heteroclinic cycle of the initial type. Therefore a sub-domain $u_{2} \subset u_{1}$ can be found, which corresponds to existence of two different Lorenz-like attractors. Repeating this procedure we obtain the required result.

Remark 2.3. Let $\mathcal{N}_{w L a} \subset \mathcal{N}$ be a subset of parameters in which the diffeomorphism $F_{\varepsilon}$ has a countable set of Lorenz attractors. Following the proof of the Theorem 2.2, the subset $\mathcal{N}_{w L a}$ is obtained as a countable intersection of open dense subsets from $\mathcal{N}$. This means that $\mathcal{N}_{w L a}$ is a subset of the second category (or a residual subset) in $\mathcal{N}$. Therefore the property of "having a countable set of coexisting Lorenz-like attractors" appears to be a typical feature of the diffeomorphisms from such Newhouse domains.

\section{On dynamical properties of the 3D Henon maps.}

In this section we pay the main attention to the problems related to the investigations of the dynamical properties of the 3D Henon map (2.6). This map can be regarded as a natural three-dimensional generalization of the well-known two-dimensional Henon map $\bar{y}=z, \quad \bar{z}=M_{1}+M_{2} y-z^{2}$; indeed the map (2.6) is quadratic, has a constant Jacobian equal to $B$ and moreover at $B=0$ it exactly becomes the 2D Henon map in coordinates $(y, z)$.

In the paper [13] (see also [14]) it was shown that

the map (2.6) possesses a (global) Lorenz-like attractor in some open domain in the parameter space.

\footnotetext{
${ }^{2}$ This attractor is local and "single-round" as it belongs to $V_{i j}$. Also it is an attractor for the first return map $T_{i j}$ only.
} 
The main idea of the proof of this statement was the verification of the conditions mentioned in [22] where it was shown that if a three-dimensional map has a fixed point with the multipliers of $(-1,-1,+1)$, its second power can be locally approximated by a flow up to arbitrary order terms; the corresponding local flow normal form in certain cases coincide with the Shimizu-Morioka system

$$
\begin{aligned}
& \dot{x}=y, \\
& \dot{y}=x(1-z)-\lambda y, \\
& \dot{z}=-\alpha z+x^{2},
\end{aligned}
$$

Regarding the map (2.6) this result is formulated as follows

Lemma 3.1. [13, 14] In the parameter space $\left(M_{1}, M_{2}, B\right)$ for a small neighbourhood of the point $(-1 / 4,1,1)$ the second iteration of the map (2.6) in some neighbourhood of one of the fixed points coincides with the time-1 map along the trajectories of the flow (3.1) up to small higher order terms. Moreover the coordinates $(x, y, z)$ and the parameters $\lambda$ and $\alpha$ can take arbitrary finite values.

Note that in [23], [24] it was established (the proof without computer assistance was performed in [25]) that the flow (3.1) possesses the Lorenz attractor for the parameters values from some open domain. Applying this result to the 3D Henon map (2.6) one can obtain (following the Lemma 3.1) that near the parameters values $\left(M_{1}, M_{2}, B\right)=(-1 / 4,1,1)$ there exists a domain in which the map possesses Lorenzlike attractors. Moreover, the numerical investigations in [13] showed that such attractors may exist in sufficiently large parameter domains and can be of a significant size.

We mention that the map (2.6) demonstrates very interesting dynamics despite it has quite a simple form. Here we emphasize its features related to the strange attractors, see [13] where the corresponding questions were addressed. However there is one more important aspect which makes the maps of the form (2.6) reasonably actual in the bifurcations theory. The fact is that the 3D Henon map (2.6) can be classified as a "homoclinic map" and has a "natural" source as it arises as a normal form of the first return map near the homoclinic tangencies ${ }^{3}$. It is well known that one- and two-dimensional quadratic maps such as the parabola map (also called as the logistic map) $\bar{y}=M_{1}-y^{2}$, standard Henon map $(\bar{x}, \bar{y})=\left(y, M_{1}-B x-y^{2}\right)$, Mirá map $(\bar{x}, \bar{y})=\left(y, M_{1}+M_{2} y-x^{2}\right)$, and also the so-called generalized Henon map $(\bar{x}, \bar{y})=\left(y, M_{1}-B x-y^{2}+\varepsilon_{1} x y+\varepsilon_{2} y^{3}\right)$, arise naturally while studying the bifurcations of quadratic homoclinic tangencies: we refer the reader here to the book [26], where much information on this subject is presented, see also [7], [27], [28], [29] and [30].

Regarding the three-dimensional maps it is known since the paper [31], see also [32], [33], that the three-dimensional map of the form

$$
\bar{x}=y, \quad \bar{y}=z, \quad \bar{z}=M_{1}+B x+M_{2} z-y^{2},
$$

is a normal form of the first return map in the case of a quadratic homoclinic tangency of the manifolds of the fixed point of the saddle-focus $(2,2)^{4}$.

\footnotetext{
${ }^{3}$ Naturally the fist return map itself is defined in a sufficiently small neighbourhood of a chosen homoclinic point for small values of the perturbation parameters. But as it often happens that for this map written in the initial coordinates and initial parameters the so-called rescaling method can be applied when the coordinates and the parameters are expanded with a large scaling factor which is proportional to the first return time $\mathcal{K}$, moreover the new coordinates and parameters can take arbitrary finite values. So that throwing away the asymptotically small terms (as $\mathcal{K} \rightarrow \infty$ ) it is possible to obtain a map of a reasonably simple form which however keeps all the dynamical properties of the initial first return map.

${ }^{4}$ Note that the map (3.2) can be regarded as an inverse map to (2.6). In this case the new parameters $\left(M_{1}, M_{2}, B\right)$ from
} (3.2) have the following relations to the old ones from (2.6):

$$
M_{1}^{\text {new }}=\frac{M_{1}}{B^{2}}, M_{2}^{\text {new }}=-\frac{M_{2}}{B}, B^{\text {new }}=\frac{1}{B} .
$$

Then, for example, according to the Lemma 3.1 we are able to state that the map (3.2) possesses a Lorenz-like repeller for the corresponding values of the parameters. 


\section{Proof of the Theorem 2.1}

It is well known ([18], [7]) that in the neighbourhood $U_{1}$ of the fixed point $O_{1}$ there exist such $C^{r}$ coordinates $(x, y)=\left(x_{1}, x_{2}, y\right)$, that the local map $T_{01}(\varepsilon):=\left.F_{\varepsilon}\right|_{U_{1}}$ for any sufficiently small $\varepsilon$ has the form:

$$
\begin{aligned}
T_{01}: & \begin{array}{l}
\bar{x} \\
\bar{y}
\end{array}=\lambda_{1} R_{\varphi} x+O\left(\|x\|^{2}|y|\right) \\
& \left.=\gamma_{1}+\|x\| y^{2}\right)
\end{aligned}
$$

where $R_{\varphi}=\left(\begin{array}{cc}\cos \varphi & -\sin \varphi \\ \sin \varphi & \cos \varphi\end{array}\right)$ is a $(2 \times 2)$ rotation matrix to the angle $\varphi$. The analogous coordinates exist also in $U_{2}$ so that the map $T_{02}(\varepsilon):=\left.F_{\varepsilon}\right|_{U_{2}}$ has the following form for any sufficintly small $\varepsilon$ :

$$
\begin{aligned}
T_{02}: & \begin{array}{l}
\bar{u} \\
\bar{v}
\end{array}=\lambda_{2} R_{\psi} u+O\left(\|u\|^{2}|v|\right) \\
& =\left(\|u\| v^{2}\right)
\end{aligned}
$$

In these coordinates the local stable and unstable manifolds of the points $O_{1}$ and $O_{2}$ have the equations of: $W_{l o c}^{s}\left(O_{1}\right):\{y=0\}, W_{l o c}^{u}\left(O_{1}\right):\{x=0\}, W_{l o c}^{s}\left(O_{2}\right):\{v=0\}, W_{l o c}^{u}\left(O_{2}\right):\{u=0\}$. At $\varepsilon=0$ we take two points $M_{1}^{-}=\left(0,0, y^{-}\right) \in U_{1}$ and $M_{2}^{+}=\left(u_{1}^{+}, u_{2}^{+}, 0\right) \in U_{2}$ of the orbit $\Gamma_{12}$ and two points $M_{2}^{-}=\left(0,0, v^{-}\right) \in U_{2}$ and $M_{1}^{+}=\left(x_{1}^{+}, x_{2}^{+}, 0\right) \in U_{1}$ of the orbit $\Gamma_{21}$. It is clear that there exist such integer $q_{1}$ and $q_{2}$ that $M_{2}^{+}=F_{0}^{q_{1}}\left(M_{1}^{-}\right)$and $M_{1}^{+}=F_{0}^{q_{2}}\left(M_{2}^{-}\right)$. Let $\Pi_{l}^{+}$and $\Pi_{l}^{-}$are some small neighbourhoods of the points $M_{l}^{+}$and $M_{l}^{-}$in $U_{l}, l=1,2$. Then the global maps $T_{12}(\varepsilon):=\left.F_{\varepsilon}^{q_{1}}\right|_{\Pi_{1}^{-}}: \Pi_{1}^{-} \rightarrow \Pi_{2}^{+}$and $T_{21}(\varepsilon):=\left.F_{\varepsilon}^{q_{2}}\right|_{\Pi_{2}^{-}}: \Pi_{2}^{-} \rightarrow \Pi_{1}^{+}$will be defined at all sufficiently small $\varepsilon$ and they can be written in the following form in coordinates (4.1)-(4.2):

$$
\begin{aligned}
& T_{12}: \bar{u}-u^{+}=A^{(1)} x+b^{(1)}\left(y-y^{-}\right)+O\left(\|x\|^{2}+\|x\| \cdot\left|y-y^{-}\right|+\left(y-y^{-}\right)^{2}\right), \\
& \bar{v}=\left(c^{(1)}\right)^{\top} x+d^{(1)}\left(y-y^{-}\right)+O\left(\|x\|^{2}+\|x\| \cdot\left|y-y^{-}\right|+\left(y-y^{-}\right)^{2}\right), \\
& T_{21}: \begin{array}{l}
\bar{x}-x^{+}=A^{(2)} u+b^{(2)}\left(v-v^{-}\right)+O\left(\|u\|^{2}+\|u\| \cdot\left|v-v^{-}\right|+\left(v-v^{-}\right)^{2}\right), \\
\bar{y}=\mu+\left(c^{(2)}\right)^{\top} u+d^{(2)}\left(v-v^{-}\right)^{2}+O\left(\|u\|^{2}+\|u\| \cdot\left|v-v^{-}\right|+\left|v-v^{-}\right|^{3}\right),
\end{array}
\end{aligned}
$$

where $d^{(1)} \neq 0$ since $W^{u}\left(O_{1}\right)$ and $W^{s}\left(O_{1}\right)$ intersect transversely at the point $M_{2}^{+}$and $d^{(2)} \neq 0$ as the tangency between $W^{u}\left(O_{2}\right)$ and $W^{s}\left(O_{1}\right)$ is quadratic.

We will also set $b^{(2)}=(b, 0)^{\top}$ and $c^{(2)}=(c, 0)$. This always can be achieved with an appropriate orthogonal rotation of the coordinates $\left(x_{1}, x_{2}\right)$ and $\left(u_{1}, u_{2}\right)$. With these rotations the form of the local maps (4.1)-(4.2) is not changed. Since $T_{12}$ and $T_{21}$ are diffeomorphisms their Jacobians $J_{12}$ and $J_{21}$ calculated in points $M_{1}^{-}$and $M_{2}^{-}$correspondingly do not vanish, i.e.

$$
J_{12}=\operatorname{det}\left(\begin{array}{ccc}
a_{11}^{(1)} & a_{12}^{(1)} & b_{1}^{(1)} \\
a_{21}^{(1)} & a_{22}^{(1)} & b_{2}^{(1)} \\
c_{1}^{(1)} & c_{2}^{(1)} & d^{(1)}
\end{array}\right) \neq 0, \quad J_{21}=\operatorname{det}\left(\begin{array}{ccc}
a_{11}^{(2)} & a_{12}^{(2)} & b \\
a_{21}^{(2)} & a_{22}^{(2)} & 0 \\
c & 0 & 0
\end{array}\right) \neq 0 .
$$

This particularly implies that

$$
b \neq 0, \quad c \neq 0, \quad a_{22}^{(2)} \neq 0 .
$$

Note that if the local maps $T_{01}$ and $T_{02}$ are given in the normal forms (4.1) and (4.2) then there exist suitable representations for their iterations, see ([7], [18]). Namely the maps $T_{01}^{k}(\varepsilon):\left(x_{0}, y_{0}\right) \rightarrow\left(x_{k}, y_{k}\right)$ for sufficiently large $k$ can be written in the following cross-form:

$$
\begin{aligned}
& x_{k}=\lambda_{1}^{k} R_{k \varphi} x_{0}+\lambda_{1}^{2 k} \rho_{1 k}\left(x_{0}, y_{k}, \varepsilon\right) \\
& y_{0}=\gamma_{1}^{-k} y_{k}+\lambda_{1}^{k} \gamma_{1}^{-k} \rho_{2 k}\left(x_{0}, y_{k}, \varepsilon\right),
\end{aligned}
$$

and analogously the maps $T_{02}^{k}(\varepsilon):\left(u_{0}, v_{0}\right) \rightarrow\left(u_{k}, v_{k}\right)$ can be represented in the form:

$$
\begin{aligned}
& u_{k}=\lambda_{2}^{k} R_{k \psi} u_{0}+\lambda_{2}^{2 k} \rho_{3 k}\left(u_{0}, v_{k}, \varepsilon\right) \\
& v_{0}=\gamma_{2}^{-k} v_{k}+\lambda_{2}^{k} \gamma_{2}^{-k} \rho_{4 k}\left(u_{0}, v_{k}, \varepsilon\right)
\end{aligned},
$$


where functions $\rho_{k}$ and their derivatives up to order $(r-2)$ are uniformly bounded in $k$.

Now, using the formulas (4.3), (4.4), (4.7) and (4.8), we obtain the following form for the first return map $T_{i j} \equiv T_{21} T_{02}^{j} T_{12} T_{01}^{i}: \Pi_{1}^{+} \rightarrow \Pi_{1}^{+}:$

$$
\begin{aligned}
& \bar{u}-u^{+}=A^{(1)} \lambda_{1}^{i} R_{i \varphi} x+b^{(1)}\left(y-y^{-}\right)+O\left(\lambda_{1}^{2 i}\|x\|^{2}+\lambda_{1}^{i}\|x\| \cdot\left|y-y^{-}\right|+\left(y-y^{-}\right)^{2}\right) \\
& \gamma_{2}^{-j}\left(\bar{v}+\lambda_{2}^{j} \rho_{4 j}\right)=\left(c^{(1)}\right)^{\top} \lambda_{1}^{i} R_{i \varphi} x+d^{(1)}\left(y-y^{-}\right)+ \\
& +O\left(\lambda_{1}^{2 i}\|x\|^{2}+\lambda_{1}^{i}\|x\| \cdot\left|y-y^{-}\right|+\left(y-y^{-}\right)^{2}\right), \\
& \bar{x}-x^{+}=A^{(2)} \lambda_{2}^{j} R_{j \psi} \bar{u}+b^{(2)}\left(\bar{v}-v^{-}\right)+O\left(\lambda_{2}^{2 j}\|\bar{u}\|^{2}+\lambda_{2}^{j}\|\bar{u}\| \cdot\left|\bar{v}-v^{-}\right|+\left(\bar{v}-v^{-}\right)^{2}\right) \\
& \gamma_{1}^{-i}\left(\bar{y}+\lambda_{1}^{i} \rho_{2 i}\right)=\mu+\left(c^{(2)}\right)^{\top} \lambda_{2}^{j} R_{j \psi} \bar{u}+d^{(2)}\left(\bar{v}-v^{-}\right)^{2}+ \\
& +O\left(\lambda_{2}^{2 j}\|\bar{u}\|^{2}+\lambda_{2}^{j}\|\bar{u}\| \cdot\left|\bar{v}-v^{-}\right|+\left|\bar{v}-v^{-}\right|^{3}\right) .
\end{aligned}
$$

Make the following shift of coordinates:

$$
u_{\text {new }}=u-u^{+}+\alpha_{i j}^{1}, v_{\text {new }}=v-v^{-}+\alpha_{i j}^{2}, x_{\text {new }}=x-x^{+}+\alpha_{i j}^{3}, y_{\text {new }}=y-y^{-}+\alpha_{i j}^{4},
$$

where the coefficients $\alpha_{i j}=O\left(\lambda_{1}^{i}+\lambda_{2}^{j}+\gamma_{1}^{-i}+\gamma_{2}^{-j}\right)$ are chosen in the way to eliminate the constant term in the equations for $\bar{x}, \bar{u}$ and $\bar{v}$ and the linear term $\bar{v}$ in the equation for $\bar{y}$. With this shift, the coefficient at $\bar{v}$ in the equation for $\bar{x}_{2}$ may become non-zero (namely, of order $\alpha_{i j}$ ) but it vanishes again with an additional rotation of the coordinates $\left(x_{1}, x_{2}\right)$ to a corresponding small angle. The system (4.9) now takes the form

$$
\begin{aligned}
& \bar{u}=A^{(1)} \lambda_{1}^{i} R_{i \varphi} x+b^{(1)} y+O\left(\lambda_{1}^{2 i}\|x\|^{2}+\lambda_{1}^{i}\|x\| \cdot|y|+y^{2}\right), \\
& \gamma_{2}^{-j} \bar{v}\left(1+O\left(\lambda_{2}^{j}\right)\right)=\left(c^{(1)}\right)^{\top} \lambda_{1}^{i} R_{i \varphi} x+d^{(1)} y+O\left(\lambda_{1}^{2 i}\|x\|^{2}+\lambda_{1}^{i}\|x\| \cdot|y|+y^{2}\right), \\
& \bar{x}=A^{(2)} \lambda_{2}^{j} R_{j \psi} \bar{u}+b^{(2)} \bar{v}+O\left(\lambda_{2}^{2 j}\|\bar{u}\|^{2}+\lambda_{2}^{j}\|\bar{u}\| \cdot|\bar{v}|+\bar{v}^{2}\right), \\
& \gamma_{1}^{-i} \bar{y}\left(1+O\left(\lambda_{1}^{i}\right)\right)=M+\left(c^{(2)}\right)^{\top} \lambda_{2}^{j} R_{j \psi} \bar{u}+d^{(2)} \bar{v}^{2}+O\left(\lambda_{2}^{2 j}\|\bar{u}\|^{2}+\lambda_{2}^{j}\|\bar{u}\| \cdot|\bar{v}|+|\bar{v}|^{3}\right),
\end{aligned}
$$

where the new coefficients and the matrices $\left(A^{(1)}, b^{(1)}, d^{(1)}\right.$ etc.) may differ from the initial ones by the values of order $\alpha_{i j}$. Moreover,

$$
M=\mu+\lambda_{2}^{j}\left(c^{(2)}\right)^{\top} u^{+}(1+\ldots)-\gamma_{1}^{-i} y^{-}(1+\ldots),
$$

where the dots denote the terms which tend to zero as $i, j \rightarrow \infty$.

Consider the second equation from (4.10). Provided that $d^{(1)} \neq 0$, for any large $i$ and $j$ the new coordinates $y$ and $v$ can be introduced in which this equation takes a linear form of $\gamma_{2}^{-j} \bar{v}=d^{(1)} y$ (i.e. $y_{n e w}$ is the new right-hand side of the equation divided by $d^{(1)}$ and $v_{n e w}$ is the left part divided by $\gamma_{2}^{-j}$ correspondingly). Next, the change of coordinates of the form $u_{n e w}=u-\left(b^{(1)} / d^{(1)}\right) \gamma_{2}^{-j} v+O\left(\gamma_{2}^{-2 j} v^{2}\right)$ excludes from the equations for $\bar{u}$ all the terms depending on $y$ only. The system (4.10) takes the form:

$$
\begin{aligned}
& \bar{u}=A^{(1)} \lambda_{1}^{i} R_{i \varphi} x+O\left(\lambda_{1}^{2 i}\|x\|^{2}+\lambda_{1}^{i}\|x\| \cdot|y|\right), \\
& \gamma_{2}^{-j} \bar{v}=d^{(1)} y \\
& \bar{x}=A^{(2)} \lambda_{2}^{j} R_{j \psi} \bar{u}+\left(\begin{array}{c}
b \\
0
\end{array}\right) \bar{v}+O\left(\lambda_{2}^{2 j}\|\bar{u}\|^{2}+\lambda_{2}^{j}\|\bar{u}\| \cdot|\bar{v}|+\bar{v}^{2}\right), \\
& \gamma_{1}^{-i} \bar{y}\left(1+O\left(\lambda_{1}^{i}\right)\right)=M+\left(c^{(2)}\right)^{\top} \lambda_{2}^{j} R_{j \psi} \bar{u}+d^{(2)} \bar{v}^{2}+O\left(\lambda_{2}^{2 j}\|\bar{u}\|^{2}+\lambda_{2}^{j}\|\bar{u}\| \cdot|\bar{v}|+|\bar{v}|^{3}\right) .
\end{aligned}
$$

We note that the last changes of the coordinates can bring us to appearance of a linear in $\bar{v}$ term of order $\left(\lambda_{2}^{j} \gamma_{2}^{-j}\right)$ in the fourth equation. We will make it vanish again with an additional shift of coordinates $(x, y, u, v)$ by a small value of the same order so that in (4.12) this term is absent.

From the first two equations of the system (4.12) the coordinates $\bar{u}$ and $\bar{v}$ can be explicitly found in terms of $x$ and $y$. Substituting the resulting expressions to the last two equations we obtain the following 
representation of the first return map $T_{i j}:(x, y) \mapsto(\bar{x}, \bar{y})$ :

$$
\begin{aligned}
& \bar{x}_{1}=b d^{(1)} \gamma_{2}^{j} y+O\left(\lambda_{2}^{j} \lambda_{1}^{i}\|x\|+\gamma_{2}^{2 j} y^{2}\right), \\
& \bar{x}_{2}=K_{1}(i, j) \lambda_{2}^{j} \lambda_{1}^{i} x_{1}+K_{2}(i, j) \lambda_{2}^{j} \lambda_{1}^{i} x_{2}+O\left(\lambda_{2}^{j} \lambda_{1}^{2 i}\|x\|^{2}+\lambda_{2}^{j} \lambda_{1}^{i} \gamma_{2}^{j}\|x\| \cdot|y|+\gamma_{2}^{2 j} y^{2}\right), \\
& \gamma_{1}^{-i} \bar{y}\left(1+O\left(\lambda_{1}^{i}\right)\right)=M+c \lambda_{2}^{j} \lambda_{1}^{i}\left(C_{1}(i \varphi, j \psi) x_{1}+C_{2}(i \varphi, j \psi) x_{2}\right)+ \\
& +d^{(2)}\left(d^{(1)}\right)^{2} \gamma_{2}^{2 j} y^{2}+O\left(\lambda_{2}^{j} \lambda_{1}^{2 i}\|x\|^{2}+\lambda_{2}^{j} \lambda_{1}^{i} \gamma_{2}^{j}\|x\| \cdot|y|+\gamma_{2}^{3 j}|y|^{3}\right),
\end{aligned}
$$

where $K_{l}(i, j), l=1,2$ are some uniformly bounded in $i, j$ coefficients and

$$
\begin{aligned}
& C_{1}(i \varphi, j \psi)=\cos i \varphi\left(a_{11}^{(2)} \cos j \psi-a_{21}^{(2)} \sin j \psi\right)+\sin i \varphi\left(a_{12}^{(2)} \cos j \psi-a_{22}^{(2)} \sin j \psi\right), \\
& C_{2}(i \varphi, j \psi)=-\sin i \varphi\left(a_{11}^{(2)} \cos j \psi-a_{21}^{(2)} \sin j \psi\right)+\cos i \varphi\left(a_{12}^{(2)} \cos j \psi-a_{22}^{(2)} \sin j \psi\right) .
\end{aligned}
$$

Note that $C_{1}^{2}+C_{2}^{2}=\left(a_{11}^{(2)} \cos j \psi-a_{21}^{(2)} \sin j \psi\right)^{2}+\left(a_{12}^{(2)} \cos j \psi-a_{22}^{(2)} \sin j \psi\right)^{2}$. Since $a_{22}^{(2)} \neq 0$ (by means of (4.6)), $C_{1}$ and $C_{2}$, generally speaking, do not vanish simultaneously ${ }^{5}$.

Now we will consider only such integer pairs of $i$ and $j$ for which the value $J_{i j}=\left(\lambda_{1}^{2} \gamma_{1}\right)^{i}\left(\lambda_{2}^{2} \gamma_{2}\right)^{j} \equiv$ $\left(J_{1}\right)^{i}\left(J_{2}\right)^{j}$ remains uniformly bounded in $i, j$. They always exist because $\ln J_{1} \cdot \ln J_{2}<0$ by the assumption, see formula (2.5)).

Finally introduce the following coordinates (rescaling-coordinates) for (4.13):

$$
y=-\frac{\gamma_{1}^{-i} \gamma_{2}^{-2 j}}{d^{(2)}\left(d^{(1)}\right)^{2}} Z, \quad x_{1}=-\frac{b \gamma_{1}^{-i} \gamma_{2}^{-j}}{d^{(2)} d^{(1)}} Y, \quad x_{2}=-\frac{\lambda_{2}^{j} \lambda_{1}^{i} \gamma_{1}^{-i} \gamma_{2}^{-j}}{c d^{(2)}\left(d^{(1)}\right)^{2}} X
$$

Then the system (4.13) is rewritten in the form:

$$
\begin{aligned}
& \bar{Y}=Z+O\left(\lambda_{2}^{j} \lambda_{1}^{i}\right), \quad \bar{X}=b d^{(1)} K_{1}(i, j) Y+O\left(\lambda_{2}^{j} \lambda_{1}^{i}\right), \\
& \bar{Z}=M_{1}+M_{2} Y+J_{i j}\left(C_{2}(i \varphi, j \psi)+\ldots\right) X-Z^{2}+O\left(\lambda_{2}^{j} \lambda_{1}^{i}\right),
\end{aligned}
$$

where

$$
M_{1}=-d^{(2)}\left(d^{(1)}\right)^{2} \gamma_{1}^{2 i} \gamma_{2}^{2 j} M, \quad M_{2}=c\left(\lambda_{1} \gamma_{1}\right)^{i}\left(\lambda_{2} \gamma_{2}\right)^{j} C_{1}(i \varphi, j \psi) .
$$

Since the coefficient in front of $M$ in (4.17) is asymptotically large as $i, j \rightarrow \infty$, the parameter $M_{1}$ for large $i, j$ may take arbitrary finite values while varying $\mu$ near the following values where $M$ vanishes, see (4.11).

The coefficient $\left(\lambda_{1} \gamma_{1}\right)^{i}\left(\lambda_{2} \gamma_{2}\right)^{j} \equiv J_{i j} \lambda_{2}^{-j} \lambda_{1}^{-i}$ in the formula (4.17) for $M_{2}$ is asymptotically large (for such $i$ and $j$ that $J_{i j}$ is uniformly bounded). Therefore while varying $\varphi$ near the values where $C_{1}(i \varphi, j \psi)=$ 0 (such values are dense $\varphi$ on any interval within $(0, \pi)$ ) it is possible to obtain arbitrary finite values of the parameter $M_{2}$.

In the cases when all coefficients in (4.16) are uniformly bounded, the corresponding maps in the rescaling-coordinates will be asymptotically close to the Henon-like map with the Jacobian of

$$
B=b d^{(1)} K_{1}(i, j) C_{2}(i \varphi, j \psi) J_{i j}
$$

The map (4.16) is nothing else but the first return map $T_{i j}$. Its domain in the initial coordinates $\left(x_{1}, x_{2}, y\right)$ corresponds to bounded values of $(Y, X, Z)$ according to (4.15). The Jacobian $J\left(T_{i j}\right)$ of the map $T_{i j}$ can be easily calculated in the initial coordinates, [34] (when the local maps $T_{01}$ and $T_{02}$ are brought to the normal forms, (4.1) and (4.2)). Namely,

$$
J\left(T_{i j}\right)=J\left(T_{01}^{i}\right) J\left(T_{02}^{j}\right) J\left(T_{12}\right) J\left(T_{21}\right)=J_{i j} J\left(T_{12}\left(M_{1}^{-}\right)\right) J\left(T_{21}\left(M_{2}^{-}\right)\right)(1+\ldots) .
$$

Note that all the changes of coordinates performed to bring the map $T_{i j}$ to the form (4.16) were linear or changes close to identity as $i, j \rightarrow \infty$. Therefore the Jacobian of the map (4.16) coincides

\footnotetext{
${ }^{5}$ Excluding the case when $a_{11}^{(2)} a_{22}^{(2)}-a_{21}^{(2)} a_{12}^{(2)}=0$ which means that $\tan j \psi=a_{12}^{(2)} / a_{22}^{(2)}$. Nevertheless even in this case we can choose such $j$ that $C_{1}^{2}+C_{2}^{2} \neq 0$.
} 
with that of $J\left(T_{i j}\right)$ up to asymptotically small terms as $i, j \rightarrow+\infty$. So that $b d^{(1)} K_{1}(i, j) C_{2}(i \varphi, j \psi)=$ $J\left(T_{12}\left(M_{1}^{-}\right)\right) J\left(T_{21}\left(M_{2}^{-}\right)\right)(1+\ldots)$ and

$$
B=J\left(T_{12}\left(M_{1}^{-}\right)\right) J\left(T_{21}\left(M_{2}^{-}\right)\right)\left(\lambda_{1}^{2} \gamma_{1}\right)^{i}\left(\lambda_{2}^{2} \gamma_{2}\right)^{j}
$$

Finally to complete the proof of the Theorem 2.1, perform a change of $X$ to $b d^{(1)} K_{1}(i, j) X_{n e w}$, after which the map (4.16) takes the required form (2.2).

Acknowledgements. The paper has been supported by the grants of RFBR No.11-01-00001, 13-01-00589 and 13-01-97028-povolzhje, by FCP "Cadry" No.14.B37.21.0361, Russian Federal Program "Scientific and scientificeducational brain-power of innovative Russia" (Contract No. 14.B37.21.0863) and the Leverhulme Trust grant RPG-279.

\section{References}

[1] S.V. Gonchenko, O.V. Sten'kin, L.P. Shilnikov. On existence of infinitely many stable and unstable invariant tori for systems from newhouse regions with heteroclinic tangencies. Rus. Nonlinear Dynamics, 2 (2006), 3-25.

[2] N.K. Gavrilov. On three-dimensional dynamical systems having a structurally unstable homoclinic contour. Rus. Math. Notes, 14 (1973), 687-696.

[3] N.K. Gavrilov, L.P. Shilnikov. On three-dimensional dynamical systems close to systems with a structurally unstable homoclinic curve. I. Math. USSR Sbornik, 17 (1972), 467-485; II. Math. USSR Sbornik, 19 (1973), $139-156$.

[4] S.V. Gonchenko, L.P. Shilnikov, D.V. Turaev. On Newhouse regions of two-dimensional diffeomorphisms close to a diffeomorphism with a nontransversal heteroclinic cycle. Proc. Steklov Inst. Math. 216 (1997), 70-118.

[5] S.V. Gonchenko, L.P. Shilnikov, O.V. Stenkin. On Newhouse regions with infinitely many stable and unstable invariant tori. Proceedings of the Int. Conf. "Progress in Nonlinear Science" dedicated to 100th Anniversary of A.A. Andronov, July 2-6; v. 1 "Mathematical Problems of Nonlinear Dynamics", Nizhny Novgorod (2002) 80-102.

[6] S.Gonchenko, L.Shilnikov, D.Turaev. Homoclinic tangencies of arbitrarily high orders in conservative and dissipative two-dimensional maps. Nonlinearity, 20 (2007), 241-275.

[7] S.V. Gonchenko, L.P. Shilnikov, D.V. Turaev. On dynamical properties of multidimensional diffeomorphisms from Newhouse regions. I. Nonlinearity, 21 (2008), 923-972.

[8] J.S.W. Lamb, O.V. Sten'kin. Newhouse regions for reversible systems with infinitely many stable, unstable and elliptic periodic orbits. Nonlinearity, 17 (2004), 1217-1244.

[9] A. Delshams, S.V. Gonchenko, V.S. Gonchenko, J.T. Lazaro, O. Sten'kin. Abundance of attracting, repelling and elliptic periodic orbits in two-dimensional reversible maps. Nonlinearity, 26 (2013), 1-33.

[10] S.V. Gonchenko, A.S. Gonchenko, A.O. Kazakov On new aspects of chaotic dynamics of "celtic stone". Rus. Nonlinear Dynamics, 8 (2013), 507-518.

[11] D.V. Turaev On dimension of non-local bifurcational problems. Bifurcation and Chaos, 6 (1996), 919-948.

[12] S.V. Gonchenko, L. Shilnikov, D. Turaev. On global bifurcations in three-dimensional diffeomorphisms leading to wild Lorenz-like attractors. Regul. Chaotic Dyn., 14 (2009), 137-147.

[13] S.V. Gonchenko, I.I. Ovsyannikov, C. Simó, D. Turaev. Three-dimensional Hénon-like maps and wild Lorenz-like attractors. Bifurc. Chaos, 15 (2005), 3493-3508.

[14] S.V. Gonchenko, J.D. Meiss, I.I. Ovsyannikov. Chaotic dynamics of three-dimensional Hénon maps that originate from a homoclinic bifurcation. Regular Chaotic Dyn., 11 (2006), 191-212.

[15] D.V. Turaev, L.P. Shilnikov. An example of a wild strange attractor. Sbornik Mathematics, 189 (1998), $137-160$.

[16] D.V. Turaev, L.P. Shilnikov. Pseudo-hyperbolisity and the problem on periodic perturbations of Lorenz-like attractors. Russian Dokl. Math., 77 (2008), 17-21.

[17] S.V. Gonchenko, A.S. Gonchenko, I.I. Ovsyannikov, D.V. Turaev Examples of Lorenz-like attractors in Hénon-like maps. Math. Model. Nat. Phenom. 8 (2013), 32-54.

[18] L.P. Shilnikov, A.L. Shilnikov, D.V. Turaev, L.O. Chua. Methods of qualitative theory in nonlinear dynamics. Part I, World Scientific, 1998.

[19] S.V. Gonchenko, L.P. Shilnikov. Invariants of $\Omega$-conjugacy of diffeomorphisms with a nongeneric homoclinic trajectory. Ukrainian Mathematical Journal, 42 (1990), 134-140.

[20] S.V. Gonchenko Dynamics and moduli of $\Omega$-conjugacy of $4 D$-diffeomorphisms with a structurally unstable homoclinic orbit to a saddle-focus fixed point. AMS Transl. Math., 200 (2000), 107-134.

[21] S.V. Gonchenko, L.P. Shilnikov, D.V. Turaev. On the existence of Newhouse regions near systems with non-rough Poincare homoclinic curve (multidimensional case). Russian Acad. Sci. Dokl. Math., 47 (1993), 268-283.

[22] A.L. Shilnikov, L.P. Shilnikov, D.V. Turaev. Normal forms and Lorenz attractors. Bifurc. Chaos, 3 (1993), $1123-1139$.

[23] A.L. Shilnikov. Bifurcation and chaos in the Marioka-Shimizu system. Methods of qualitative theory of differential equations, Gorky (1986), 180-193 [English translation in Selecta Math. Soviet., 10 (1991), 105-117]

[24] A.L. Shilnikov. On bifurcations of the Lorenz attractor in the Shimuizu-Morioka model. Physica D, 62 (1993), $338-346$. 
[25] G.Tigan, D.Turaev. Analytical search for homoclinic bifurcations in the Shimizu-Morioka model. Physica D: Nonlinear Phenomena, 240 (2011), 985-989.

[26] Homoclinic tangencies, edited by S.V. Gonchenko and L.P. Shilnikov, Moscow-Izhevsk, 2007.

[27] S.V. Gonchenko, V.S.Gonchenko, L.P.Shilnikov. On homoclinic origin of Henon-like maps. Regular and Chaotic Dynamics, 4-5 (2010), 462-481.

[28] S.V. Gonchenko, I.I. Ovsyannikov, D.V. Turaev. On the effect of invisibility of stable periodic orbits at homoclinic bifurcations. Physica D, 241 (2012), 1115-1122.

[29] V. Rom-Kedar, D. Turaev. Big islands in dispersing billiard-like potential. Physica D, 130 (1999), $187-210$.

[30] S.V. Gonchenko, V.S.Gonchenko. On bifurcations of the birth of closed invariant curves in the case of two-dimensional diffeomorphisms with homoclinic tangencies. Proc. Steklov Inst. Math., 244 (2004), 80-105.

[31] S.V. Gonchenko, D.V. Turaev, L.P. Shilnikov. Dynamical phenomena in multi-dimensional systems with a non-rough Poincare homoclinic curve. Russ. Acad. Sci. Dokl. Math., 47 (1993), 410-415.

[32] S.V. Gonchenko, L.P. Shilnikov, D.V. Turaev. Dynamical phenomena in systems with structurally unstable Poincare homoclinic orbits. Chaos, 6 (1996), 15-31.

[33] S.V. Gonchenko, L.P. Shilnikov, D.V. Turaev. On dynamical properties of diffeomorphisms with homoclinic tangencies. J. Math. Sci., 126 (2005), 1317-1343.

[34] S.V. Gonchenko, V.S. Gonchenko, J.C. Tatjer. Bifurcations of three-dimensional diffeomorphisms with non-simple quadratic homoclinic tangencies and generalized Hénon maps. Regular and Chaotic Dynamics, 12 (2007), 233-266. 